\title{
A119 IL-32 $\gamma$ AND STREPTOCOCCUS PYOGENES CELL WALL FRAGMENTS SYNERGISE FOR IL-1-DEPENDENT DESTRUCTIVE ARTHRITIS VIA UPREGULATION OF TLR-2 AND NOD2
}

B Heinhuis, ${ }^{1}$ M I Koenders, ${ }^{1}$ F A van de Loo, ${ }^{1}$ P L E M van Lent, ${ }^{1}$ S-H Kim, ${ }^{3}$ C A Dinarello, ${ }^{3}$ L A B Joosten, ${ }^{2}$ W B van den Berg ${ }^{1}{ }^{1}$ Rheumatology Research and Advanced Therapeutics, Department of Rheumatology, Radboud University Nijmegen Medical Centre, Nijmegen, The Netherlands; ${ }^{2}$ Department of Medicine, Radboud University Nijmegen Medical Centre, Nijmegen, The Netherlands; ${ }^{3}$ Division of Infectious Diseases, University of Colorado, Denver, Colorado, USA

\subsection{6/ard.2010.129635k}

Purpose Interleukin 32 (IL-32) might play an important role in the pathogenesis of RA by inducing cytokines and chemokines. IL-32 is highly expressed in RA synovial tissue and strongly correlates with synovial inflammation, tumour necrosis factor (TNF) $\alpha$ and IL-1 expression. IL-32 alone is not that potent, but it appears to enhance sensitivity of synovial cells to proinflammatory stimuli. Here, we investigated potential synergistic effects of IL-32 $\gamma$ with Streptococcus pyogenes cell wall fragments, which contain predominantly TLR2/NOD2 ligands, in human fibroblast-like synoviocytes (FLS). In addition, we explored the synergistic arthritogenicity of IL-32 $\gamma$ and SCW fragments in wildtype and IL-1 deficient mice.

Method FLS from 10 arthritis patients were isolated and transduced with an adenoviral vector encoding for IL-32 $\gamma$ (AdIL-32 $\gamma$ ) followed by SCW stimulation. To investigate in vivo synergy, we injected AdIL-32 $\gamma$ virus together with SCW fragments intra-articularly into knee joints of wildtype and IL-1 deficient mice.

Results In human FLS synergistic upregulation of the proinflammatory cytokines IL- $1 \beta$, IL- 6 and TNF $\alpha$ was found and similar observation was made for the chemokines CCL2, CCL20 and CXCL8. Of great importance, we also identified synergistic upregulation of matrix degrading enzymes, including MMP1 and MMP3, linking the IL-32 synergy to erosive processes. Part of the synergy may be related to the marked increase of the pattern recognition receptors TLR2 and NOD2. In wildtype mice we observed a prolonged and severe arthritis injected with AdIL-32 $\gamma / \mathrm{SCW}$ compared to AdControl/SCW. Furthermore, histological analysis showed apart from enhanced numbers of inflammatory cells, severe cartilage proteoglycan depletion, and irreversible chondrocyte death in mice exposed to IL-32 $\gamma$ and TLR2/NOD2 ligands. Remarkably, IL-1 deficient mice injected with AdIL-32 $\gamma /$ SCW were completely protected against the destructive arthritis observed in wildtype mice.

Conclusions The observed synergistic effect between IL-32 $\gamma$ and TLR2/NOD2 ligands identifies an important amplifying pathway in the development of destructive arthritis which 


\section{EWRR abstracts}

is IL-1 dependent via the upregulation of TLR-2 and NOD2 receptors. Targeting of IL-32 $\gamma$ may provide a novel therapy to prevent destructive arthritis. 